\title{
Capacity Enhancement for CDMA Systems Through Adaptive Cell Sectorization
}

\author{
Cem U. Saraydar \\ Aylin Yener \\ Wireless Information Network Laboratory (WINLAB), Rutgers University \\ saraydar@winlab.rutgers.edu yener@winlab.rutgers.edu
}

\begin{abstract}
We consider the problem of adaptive cell sectorization in a CDMA system. Specifically, given the user distribution in a cell, we investigate the two problems of how to appropriately sectorize the cell such that we minimize the total received power and the total transmit power spent by all the users, in both cases, while giving each user acceptable quality of service. The optimum arrangement equalizes the number of users in each sector for the received power optimization problem. The transmit power optimization can be formulated as a graph partitioning problem which can be solved in polynomial time. In both cases, the optimum arrangement can be quite different from uniform cell sectorization (equal partitioning) under non-uniform traffic conditions. We observe that, with adaptive sectorization, received and transmit power savings are achieved and number of users served by the system (system capacity) is increased compared to uniform sectorization of the cell.
\end{abstract}

\section{Introduction}

Due to the ever increasing demand for wireless services, efficient use of resources to maximize capacity remains of utmost importance for system design. Among the ambitious goals of the future wireless systems are high quality service and coverage for maximum number of subscribers. CDMA technology emerges as a promising candidate to fulfill these goals.

It is well known that minimizing interference using power control enhances the capacity of a CDMA system [1,2]. Further, cells can be sectorized using directional antennas to introduce orthogonalization to the system, thereby increase the capacity [3]. It is however important to design the sectors such that the maximum capacity gain can be achieved under possibly highly non-uniform traffic loads. Recently, fixed wireless systems, Wireless Local Loops (WLL), are posed as an alternative to conventional local wired telephone service [4]. Contrary to mobile macro-cellular systems, for fixed wireless systems (or systems with low mobility), users whereabouts, and thus their uplink gains to the base station, do not change much with time and the information about the traffic load as a function of the position in the cell can be obtained at the base station. It is then possible to use this information to sectorize the cells such that the maximum coverage is achieved, i.e. the maximum number of subscribers get their required quality of service.

In this paper, we investigate the minimization of the total power spent in a cell. Given the number of sectors, we study the best way of sectorizing the cell such that all subscribers in the cell get their quality of service requirements while using as little power as possible. We consider the minimization of the total received power in the cell as well as the total transmit power. We show that in the received power optimization case, the best arrangement is such that the number of users in each sector is equalized (as much as possible) leading to a discrete waterfilling solution. For the minimum total transmit power problem, the solution is found by solving a shortest path problem on a network of nodes obtained from terminal locations. We provide performance comparisons of solutions by both the received power and transmit power problems with the equal sectors configuration implemented in current systems.

\section{Model}

We consider a single cell DS-CDMA system with processing gain $C$ and a total of $M$ users. The user (call traffic) density in the cell is a known function of the distance and angle (referenced to the origin) from the base station. In a WLL, this information is readily available through the addresses of the subscribers. Note that the user density can be highly nonuniform in which case partitioning the cell into equal width sectors as is done conventionally may not be the best way of maximizing the number of users to be served by the cell (capacity). We assume the cell is to be partitioned into $N$ sectors and the antennas have ideal directivity, i.e. there is no interference between the sectors.

Our aim is to find the best sectorization arrangement such that all users get their required quality of service while expending as little power as possible. In each sector, all users belonging to that sector interfere with each other. We assume, each user has a pseudo-random signature sequence and matched filters are employed at the base station. The quality of service measure is the signal to interference ratio (SIR); a user has reliable communication if its SIR at its assigned sector antenna is larger than a target $\operatorname{SIR}\left(\gamma^{*}\right)$.

\section{Received Power Optimization}

The first optimization problem we consider is minimization of total received power expended in the cell which can be ex- 
pressed as

$$
\begin{array}{ll}
\min _{\theta, \mathbf{p}} & \sum_{i=1}^{N} \sum_{j \in s_{i}(\theta)} p_{i} h_{j} \quad \text { (RP) } \\
\text { s.t. } & \gamma_{i}=\frac{p_{j} h_{j}}{\sum_{k \neq j . k \in s_{i}(\theta)} p_{k} h_{k} / G+\sigma^{2}} \geq \gamma^{*} \\
& j=1, \ldots, M \quad i=1, \ldots, N \\
& \mathbf{p} \geq \mathbf{0} \quad \mathbf{1}^{T} \theta=2 \pi
\end{array}
$$

where $\gamma_{j}, p_{j}$ and $h_{j}$ are the SIR, the transmit power and the uplink gain of the $j^{\text {th }}$ user, $s_{i}(\theta)$ is the set of users that reside in the area spanned by sector $i, \theta$ is the $N$-tuple vector that denotes the sector angles, $\mathbf{0}$ and $\mathbf{1}$ denotes the all zero and all 1 vectors, respectively. Note that the constraints represent each user's reliable connection requirement.

It is not hard to see that the constraints ought to be satisfied with equality for received or transmit power minimization. Note also that within each sector, minimum power can be achieved via assigning equal received powers to all users belonging to the sector. This is due to the fact that users have equal square correlation with each other and is true for both received and transmit power optimization.

Given the above observations, we can express the received power for sector $i, q_{i}=p_{i} h_{j}$ for all users $j$ in sector $i$, as

$$
q_{i}^{*}=\frac{\gamma^{*} \sigma^{2}}{1-\frac{\gamma^{*} M_{i}(\theta)}{G}}
$$

where $M_{i}(\theta)=\left|s_{i}(\theta)\right|$ is the number of users in sector $i$, i.e. the cardinality of set $s_{i}(\theta)$. Given (2), we can rewrite (1) as:

$$
\begin{array}{ll}
\min _{\theta . \mathbf{q}^{*}} & \sum_{i=1}^{N} M_{i}(\theta) q_{i}^{*} \\
\text { s.t. } & q_{i}^{*}=\frac{\gamma^{*} \sigma^{2}}{1-\frac{\gamma^{*} M_{i}(\theta)}{G}} \quad i=1, \ldots, N \\
& \mathbf{q}^{*} \geq \mathbf{0} \quad \sum_{i=1}^{N} M_{i}(\theta)=M \quad \mathbf{1}^{T} \theta=2 \pi
\end{array}
$$

where $\mathbf{q}^{*}$ is the $N$-vector that contains the received power values in each sector. (3) is equivalent to

$$
\begin{aligned}
& \min _{\theta} \sum_{i=1}^{N} \frac{\gamma^{*} \sigma^{2} M_{i}(\theta)}{1-\frac{\gamma^{*} M_{i}(\theta)}{G}} \quad i=1, \ldots, N \\
& M_{i}(\theta)<G / \gamma^{*} \quad \sum_{i=1}^{N} M_{i}(\theta)=M \quad \mathbf{1}^{T} \theta=2 \pi
\end{aligned}
$$

Substituting $M_{N}(\theta)=M-\sum_{i=1}^{N-1} M_{i}(\theta)$ and taking the derivatives reveals that the optimum point has to satisfy

$$
\frac{1}{1-\frac{\gamma_{G}^{*}}{G} M_{j}(\theta)}=\frac{1}{1-\frac{\gamma^{*}}{G}\left(M-\sum_{i=1}^{N-1} M_{i}(\theta)\right)}
$$

for all $j=1, \cdots, N$. If we assume $M=k N$ where $k$ is an integer, then (5) implies that the sectors should be arranged, i.e. $\theta$ should be determined, such that

$$
M_{j}(\theta)=M / N \quad j=1, \ldots, N
$$

Thus, to minimize the total received power expended in the cell while all users get their minimum required quality of service, the cell should be sectored such that each sector has the same number of active users. Clearly, any set of $\theta$ for which each sector covers $M / N$ users is optimum. So, starting from one reference point, the sectorization can be arranged such that each sector is terminated when a coverage of $M / N$ users is reached.

Note that the generalization of the result to $M / N$ not an integer is immediate in the sense that the left over users after $k=\lfloor M / N\rfloor$ are placed in each sector, should be distributed evenly to the sectors. Thus, as a result, some sectors have to serve $k+1$ users while some serve $k$. The total interference is placed as equally as possible which is the discrete water-filling solution.

\section{Transmit Power Optimization}

\subsection{Problem Statement}

Transmit power optimization problem can be stated as

$$
\begin{array}{ll}
\min _{\theta . \mathbf{p}} & \sum_{i=1}^{N} \sum_{j \in s_{i}(\theta)} p_{j} \quad \text { (TP) } \\
\text { s.t. } & \gamma_{j}=\frac{p_{j} h_{j}}{\sum_{k \neq j . k \in s_{i}(\theta)} p_{k} h_{k} / G+\sigma^{2}} \geq \gamma^{*} \\
& j=1 \ldots . M \quad i=1, \ldots . N \\
& \mathbf{p} \geq \mathbf{0} \quad \mathbf{1}^{T} \theta=2 \pi
\end{array}
$$

The observations that led to (2) hold for (7). Thus, we can rewrite (7) as

$$
\begin{array}{ll}
\min _{\theta . \mathbf{q}^{*}} & \sum_{i=1}^{N} q_{i}^{*} \sum_{j \in s_{i}(\theta)} \frac{1}{h_{j}} \\
\text { s.t. } & q_{i}^{*}=\frac{\gamma \cdot \sigma^{2}}{1-\frac{\gamma^{*} M_{i}(\theta)}{(i}} \quad i=1 \ldots \ldots N \\
& \mathbf{q}^{*} \geq \mathbf{0} \quad \sum_{i=1}^{N} M_{i}(\theta)=M \quad \mathbf{1}^{T} \theta=2 \pi
\end{array}
$$

which is equivalent to

$$
\begin{array}{ll}
\min _{\theta . \mathbf{q}^{*}} & \sum_{i=1}^{N} \frac{\gamma^{*} \sigma^{2}}{1-\frac{\gamma^{*} M_{i}(\theta)}{G}} \sum_{j \in s_{i}(\theta)} \frac{1}{h_{j}} \\
& \mathbf{q}^{*} \geq \mathbf{0} \quad \sum_{i=1}^{N} M_{i}(\theta)=M \quad \mathbf{1}^{T} \theta=2 \pi
\end{array}
$$


We observe that (9) can be formulated as a graph partitioning (GP) problem. We also observe that because of the special topology of the problem at hand, this problem is polynomially solvable $[5,6]$. We will first discuss how the physical wireless system entities can be represented in a graph setting. The equivalent graph theoretic problem will then be defined and solutions will be presented. Finally, we will give an algorithm that solves the TP problem.

\subsection{Graph Theoretic Formulation of TP}

TP does not have a closed form solution. However, through appropriate transformations, it can be formulated as a graph partitioning problem for which we present an algorithm that is guaranteed to find the optimal solution in polynomial time.

First, identify all the terminals within a cell by assigning an angular and radial coordinate to each one of them. The radial position is the distance of the terminal to the base station. The angular position is the angular distance of the terminal to a reference terminal. Without loss of generality, the reference terminal can be selected arbitrarily and assigned 0 -degrees as its angular position. Each terminal within the cell is represented by a vertex (node) along a ring. Let the position of the vertex on the ring be determined by the angular position of the terminal regardless of its radial position. The radius of the ring is independent of the radial position of the users. Multiple users sharing exactly the same angular position will be denoted by vertices positioned along a path that protrudes from the ring at this angular position. If there exist such terminals, the ring will be modified to eliminate the multi-node extension of the ring by combining these vertices into a super-node. This reduction can be done with no effect on the optimal sectorization outcome since it is physically not possible to place users sharing the same angular position into different sectors. Figure 1 demonstrates the relation between the physical location of terminals and the corresponding nodes along a ring.

The topology just described constitute the elements of an undirected connected graph, $G=(V, E)$, with $M=|V|$ vertices. The vertex set and the edge set are $V=\left\{v_{1}, v_{2}, \cdots, v_{M}\right\}$ and $E=\left\{\left(v_{i}, v_{i+1}\right) \mid i=1, \cdots, M-1\right\} \cup\left\{\left(v_{M}, v_{1}\right)\right\}$, respectively. Denote element $\left(v_{i}, v_{j}\right)$ of the edge set by $e_{i}$. Let the weight of vertex $v_{i}$ denoted by $w_{i}$. Vertex $i$ is assigned weight $w_{i}=\frac{1}{h_{i}}$ where $h_{i}$ is the uplink gain of the $i$ th terminal. The weight of a super-node is the summation of the weights of the contributing original nodes. In an undirected graph, all edges are symmetric with respect to the vertices the edge lies between. In a connected graph, there exists a sequence of edges that connects any given pair of vertices; there are no isolated nodes $[6-8]$.

\subsection{Optimal Connected Partition on a Ring}

Sectorization problem for minimum transmitted power (TP) solves for an optimal set of sector angles that identify sector boundaries. The set of terminals in the cell are opti- mally grouped into disjoint subsets. Grapt theoretic methods emerge as powerful tools to find the best partition.

Let $\pi=\left\{S_{1}, \cdots, S_{N}\right\}$ be a partition of " into $N$ subsets. $\pi$ is considered feasible in $G$ if for every $k \in\{1, \cdots, N\}$, the subgraph $G\left(S_{k}\right)$ induced by $S_{k}$ is connected and the weight $W\left(S_{k}\right)$ of $S_{k}$ as defined below is greater than or equal to zero. The set of all such feasible partitions is denoted by $\Pi(G, N)$. Note that in a feasible partition, $S_{k} \neq 0$ for all $k$.

The graph partitioning problem (GP) is to find a feasible partition such that some given cost is minimized. Mathematically, it can be expressed as,

$$
\min _{\pi \in \Pi(G, N)} C(\pi) \quad \text { (GP) }
$$

where the cost function is given as $C: \Pi(G, N) \rightarrow \mathfrak{R}$. We define the weight of a subset $S$ of $V(S \subset V)$ by

$$
W(S)=\frac{\gamma^{*} \sigma^{2}}{1-\frac{\gamma|S|}{G}} \sum_{k: v_{k} \in S} w_{k}
$$

where $|S|$ is the number of vertices in $S$. The cost of partition $\pi=\left\{S_{1}, \cdots, S_{N}\right\}$ is therefore

$$
C(\pi)=\sum_{i=1}^{N} W\left(S_{i}\right)
$$

The graph partitioning problem can now be expressed as,

$$
\min _{\left\{S_{1}, \cdots, S_{N}\right\} \in \Pi(G . N)} \sum_{i=1}^{N} W\left(S_{i}\right) \quad \text { (GP) }
$$

Note that the structure of the cost functions for GP and $\mathrm{TP}$ are the same. In addition, each feasible partition $\pi$ corresponds to a set of cell sectorization configuration $\left\{s_{i}(\theta)\right\}$, each of which have the same cost value. Therefore, it follows that a solution for GP is also a solution for TP. Notice that the graph theoretic formulation returns the optimal sectors in terms of a list of terminals that belong to each sector. However, it does not say anything about where the actual sector boundary is located. The choice of the actual sector boundary is flexible since it can lie anywhere between the closest two terminals that belong to different sectors. The location of the boundary between neighboring vertices might have practical significance, however it has no impact on the cost function and the optimization given in (13).

\subsection{Partitioning and Shortest Path Algorithms}

We show that the graph partitioning problem GP and therefore TP is polynomially solvable. We first observe that the cost function of GP given in (13) is separable.

Definition 1 A function of $M$ variables $f\left(x_{1}, x_{2}, \cdots, x_{M}\right)$ is said to be separable if it can be expressed as a sum of $M$ functions of a single variable; i.e. $f\left(x_{1}, x_{2}, \cdots, x_{M}\right)=\sum_{i=1}^{M} f_{i}\left(x_{i}\right)$.

We use the following optimal graph partitioning result presented in $|5,6|$. 
Theorem 1 If the objective function is separable, the problem of optimally partitioning a string, which is a special graph that can be obtained by placing all the vertices along a line, can be reduced to a shortest path problem with computational complexity $\left.Q M^{2} N\right)$.

Proof can be found in [9]. Although the cost function in (13) is separable, the graph is a ring instead of the string that Theorem 1 applies for. With some increase in computational complexity, we can modify GP so that optimal partition can be found by solving the corresponding shortest path problem. When disconnected at some arbitrary edge, the ring is transformed into a string. We can then use a shortest path algorithm to solve for the best connected $N$ partition with complexity $\left.Q M^{2} N\right)$. Repeating the same procedure for each of the $M$ edges in $E$, and choosing the best among these solutions we end up with the optimal solution for GP with computational complexity $\left.Q M^{3} N\right)$. It is perhaps possible to do better than $\left.Q M^{3} N\right)$ however this is a topic beyond the scope of this work.

Algorithm to find the partition that solves TP can be given as follows.

1. Given the location of the terminals, construct a graph as described in Section 4.2.

2. For each edge in the graph:

(a) Remove the edge to obtain a string.

(b) Find optimal partitioning of the string as described in Section 4.4. Store the optimal configuration and the corresponding cost.

3. Compare the costs and choose the partition corresponding to the minimum cost.

The partition returned by this algorithm solves GP and therefore TP. The sectors are then formed based on this optimal partition.

\section{Results and Discussion}

We provide sectorization results by three methods: transmit power optimization, received power optimization, and uniform sectorization. The output of the algorithm given in Section 4.4 is the optimal partitioning (OP) that minimizes sum of transmit powers of all users in the cell. Sectorization that places equal number of terminals in each sector (EP) is the minimum received power result. Finally, in uniform sectorization (UP), sectors are of equal width and their orientation does not depend on the terminal locations.

Figure 2 shows sector boundaries obtained by OP and EP. For this particular example, UP is not feasible, i.e. the system cannot support acceptable SIR to all users in the cell if uniform sectorization of the cell is employed. The adaptive sectorization enables the system to support all users. Recall that OP has complexity $\left.Q M^{3} N\right)$. Since EP is much easier to implement, one might wonder how EP performs in terms of total

\begin{tabular}{|c|c|c|}
\hline & Figure 2 & Figure 3 \\
\hline TTP(OP) & 6.3 & 3.9 \\
\hline TTP(EP) & 9.1 & 4.2 \\
\hline TTP(UP) & Infeasible & 5.8 \\
\hline
\end{tabular}

Table 1: Total transmit powers [Watts] obtained from three methods.

transmit power. We have compared the total transmit power values for all three methods in Table 1. It can be seen that, for this example, OP and EP result in quite different total transmit power values and OP brings $40 \%$ improvement in total transmit power (TTP) as compared to EP. This is an example where finding OP really pays off in terms of transmit power savings.

Figure 3 shows a scenario where the distribution of the terminals are uniform in area within the cell. In this example, TTP(EP) reduces by $6 \%$ and $\mathrm{TTP}(\mathrm{UP})$ reduces by $30 \%$ as a result of optimal partitioning (Table 1). In general, in case of uniformly distributed terminals, TTPs with OP, EP and UP are expected to be close to each other. An extreme case where all three methods yield the same TTP is when the terminals lie along the perimeter of a circle at uniform angular spacing.

In this paper, we studied the joint total power optimization and adaptive sectorization problem for both the received and transmit power optimization cases. It is shown that the total received power is minimized by equalizing the number of users in each sector (EP) while the transmit power optimizing partition (OP) can be found with polynomial complexity using graph theory approach. OP is expected to reduce total transmit power the most in scenarios similar to the one given in Figure 2 where terminals are not distributed uniformly around the cell.

Acknowledgement The authors would like to thank Dr. Bruno Simeone and Dr. Tonguc Unluyurt for helpful discussions throughout this work.

\section{References}

[1] A. J. Viterbi. CDMA: Principles of Spread Spectrum Communication. Addison Wesley.

[2] R. D. Yates. A framework for uplink power control in cellular radio systems. IEEE JSAC. 13(7):1341-1347. 1995

[3] G. K. Chan. Effects of sectorization on the spectrum efficiency of cellular radio systems. IEEE Transactions on Communications. 41(3):217-225. August 1992

[4] V. K. Garg and E. L. Sneed. Digital wireless local loop system. IEEE Comm. Magazine. pages 112-115. October 1996.

[5] B. Simeone. Optimal connected partitions on graphs. Tutorial Series at DIMACS Center. Rutgers University. NJ. May 1999.

[6] C. De Simone, M. Lucertini. S. Pallottino, and B. Simeone Fair dissections of spiders. worms. and caterpillars. Networks. 20:323-344. 1990)

17) E. L. Lawler. Cobinatorial Optimization: Networks and Matroids. Holt, Rinchart and Winston. 1976. 
[8] C. Berge. Graphs. Elsevier Science Pub. Co., 1985.

[9] C. U. Saraydar and A. Yener. Adaptive cell sectorization in CDMA systems. http://www.winlab.rutgers.edu/ yener.

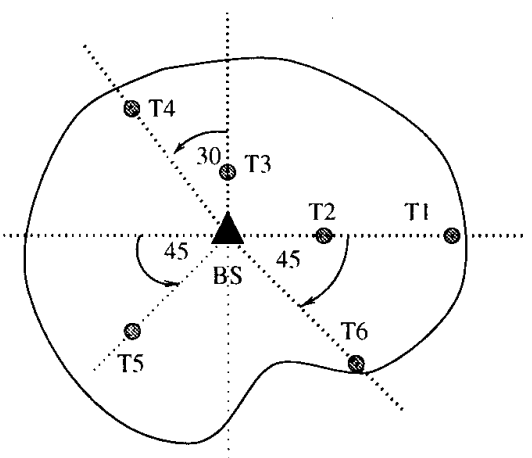

(a) Users in the cell

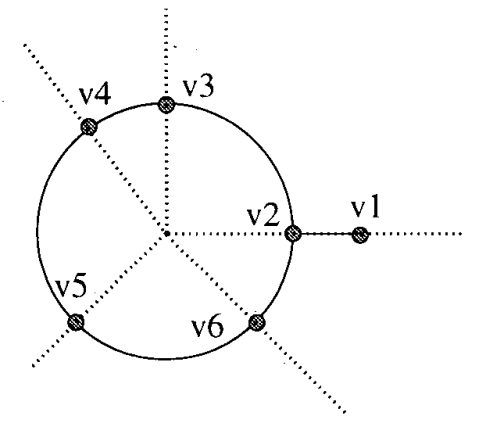

(b) Equivalent Graph

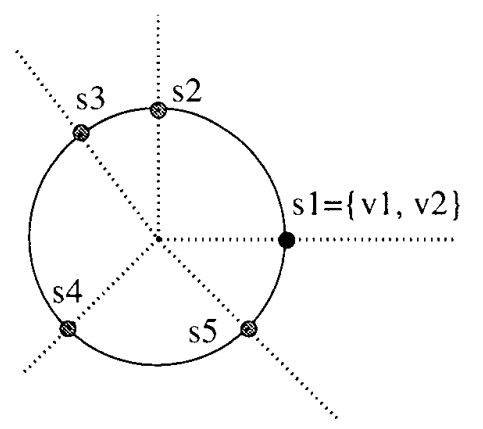

(c) Reduced Graph

Figure 1: (a) Terminal locations within a cell (b) The graph that is constructed based on the terminal locations (c) Reduced graph obtained by constructing supernodes by combining vertices sharing a common angular position.

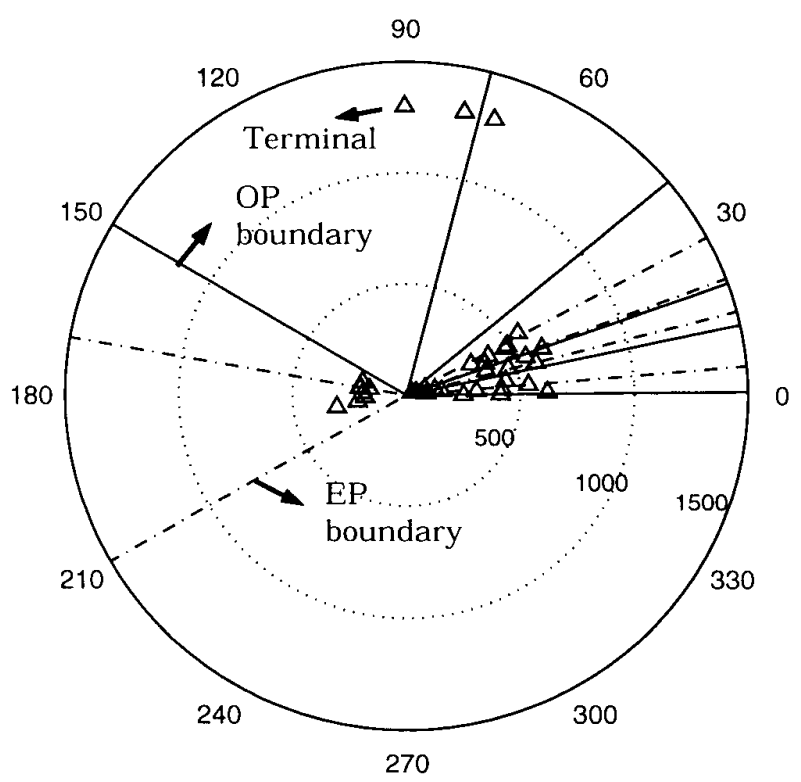

Figure 2: Sector boundaries of OP and EP in a cell where the number of terminals, $M=36$, number of sectors, $N=6$, processing gain, $G=64$, target SIR, $\gamma^{*}=7 d B$ and noise power, $\sigma^{2}=10^{-13}$.

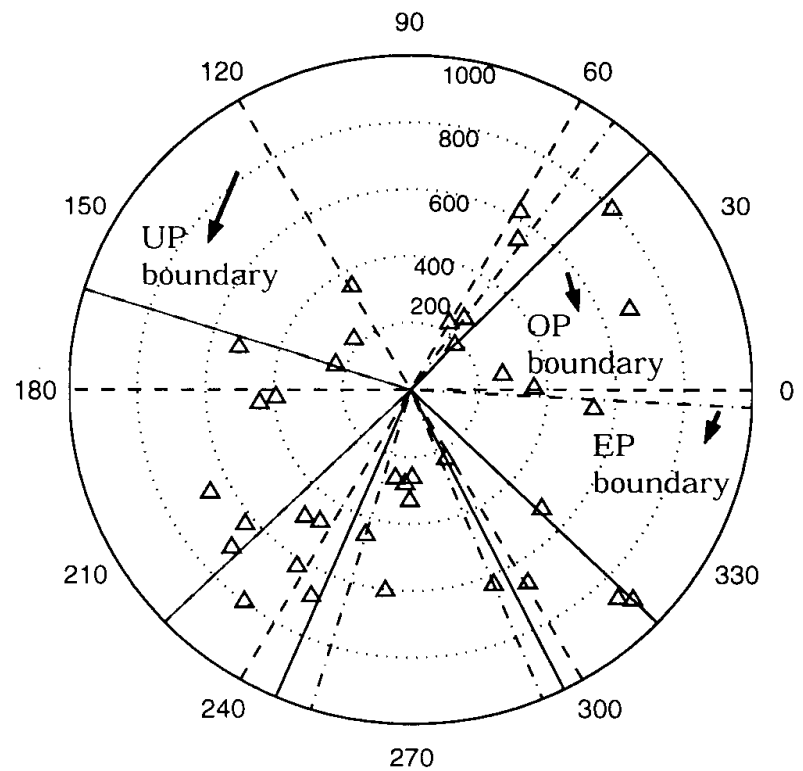

Figure 3: Sector boundaries of OP. EP and UP in a cell where the number of terminals, $M=36$, number of sectors, $N=6$, processing gain, $G=64$, target SIR, $\gamma^{*}=7 d B$ and noise power, $\sigma^{2}=10^{-13}$. The distribution of terminals is uniform in area. 